\title{
Sarcopenia and Sarcopenic Obesity Are Prognostic Factors for Overall Survival in Patients with Cirrhosis
}

\author{
Nagisa Hara ${ }^{1}$, Motoh Iwasa ${ }^{2}$, Ryosuke Sugimoto ${ }^{2}$, Rumi Mifuji-Moroka ${ }^{2}$, \\ Kyoko Yoshikawa ${ }^{2}$, Eriko Terasaka ${ }^{1}$, Ayana Hattori ${ }^{1}$, Masumi Ishidome ${ }^{1}$, \\ Yoshinao Kobayashi ${ }^{2}$, Hiroshi Hasegawa ${ }^{2}$, Kazuko Iwata ${ }^{1}$ and Yoshiyuki Takei ${ }^{2}$
}

\begin{abstract}
Objective Although the prognosis is known to be poor in cirrhosis patients associated with sarcopenia, the relationships among skeletal muscle, visceral fat, and the liver have not yet been thoroughly investigated. Therefore, the prognosis and its associations with body composition and the severity of liver disease were examined in patients with cirrhosis.

Methods The skeletal muscle mass and visceral fat area were measured in 161 patients with cirrhosis, the effects of body composition on the prognosis were analyzed, and any factors that contribute to changes in body composition were assessed.

Results During the mean observation period of 1,005 days, 73 patients died. Patients with sarcopenia or sarcopenic obesity had a poor prognosis, and this difference was pronounced in the subset of patients classified as Child-Pugh class A. A decreased skeletal muscle mass was strongly correlated with decreased serum albumin levels. Sarcopenia is a common feature of advanced cirrhosis, and transitions were observed from normal body composition to sarcopenia and from obese to sarcopenic obesity.

Conclusion The body composition is a prognostic factor for cirrhosis, and a better body composition may be advantageous for obtaining a long-term survival in patients with cirrhosis.
\end{abstract}

Key words: liver cirrhosis, prognosis, sarcopenic obesity, skeletal muscle, visceral fat

(Intern Med 55: 863-870, 2016)

(DOI: 10.2169/internalmedicine.55.5676)

\section{Introduction}

A loss of skeletal muscle mass (sarcopenia) is very frequently observed in chronic diseases such as obstructive pulmonary disease, malignant tumors, chronic rheumatoid arthritis, and cirrhosis, and when sarcopenia accompanies these diseases, the incidence of adverse events increases and the prognosis worsens (1-4). In addition, in such chronic diseases, a reduced fat mass, or undernutrition, is also known to lead to a poor prognosis (5). On the other hand, although it is a well-known fact that visceral obesity causes insulin resistance and leads to a high risk of developing cancer or cardiovascular events, it is also reported that sarco- penic obesity (the coexistence of sarcopenia and visceral obesity) is an advanced metabolic disorder showing severe metabolic syndrome $(6,7)$. Thus, the skeletal muscle mass and visceral fat mass affect the pathophysiology of a disease, and their effects differ depending on the disease (5).

A high incidence of serious events and poor outcomes have been reported when sarcopenia accompanies cirrhosis (4). In terms of body fat, it was recently reported that the prognosis is poor after resection of hepatocellular carcinoma (HCC) in cirrhosis patients with decreased visceral fat, and that the maintenance of the visceral fat mass is therefore essential to maintain a satisfactory prognosis after liver resection (8). For cirrhosis treatment, nutritional assessments are conducted, and efforts are made to improve the

${ }^{1}$ Nutrition Unit, Mie University Hospital, Japan and ${ }^{2}$ Department of Gastroenterology and Hepatology, Mie University Graduate School of Medicine, Japan

Received for publication April 28, 2015; Accepted for publication July 27, 2015

Correspondence to Dr. Motoh Iwasa, motoh@clin.medic.mie-u.ac.jp 
protein-energy malnutrition state $(9,10)$; however, there have been very few investigations related to body fat, and the optimal level of visceral fat mass has not yet been elucidated. Moreover, which test measurements are associated with the progression of sarcopenia in cirrhosis has not been evaluated, and there are also very few findings related to countermeasures for sarcopenia.

In the present study, the skeletal muscle mass and visceral fat mass of patients with cirrhosis were measured, and the relationships between these body composition measurements and the prognosis were investigated. Furthermore, timedependent changes in body composition measurements, as well as factors related to the amounts of these changes, were also examined.

\section{Materials and Methods}

A total of 161 patients with cirrhosis (94 men, 67 women; mean age 67 \pm 9 years) who received medical care and nutritional assessment at the Mie University Hospital between February 2006 and June 2013 were investigated. The diagnosis of cirrhosis was made based on imaging tests such as abdominal ultrasound, computed tomography (CT), and magnetic resonance imaging, blood test findings, and the presence of any evidence of portal hypertension, such as esophageal varices. Liver biopsies were performed for conditions that were difficult to diagnose. The coexistence of diabetes was also determined, in addition to the coexistence of HCC, and the liver function was evaluated using the Child-Pugh classification. As a general rule, nutritional guidance was provided to cirrhosis patients in accordance with the nutritional standards of the Japan Society of Metabolism and Clinical Nutrition or the European Society of Parenteral and Enteral Nutrition (ESPEN) guidelines (11). Additionally, 20-30 minutes of aerobic exercise 2-3 times a week was recommended in patients with compensated cirrhosis.

Serum albumin, alanine aminotransferase, and total bilirubin were measured as blood tests. Regarding body composition, in addition to height and weight, the extracellular water (ECW) ratio, visceral fat area $\left(\mathrm{VFA}, \mathrm{cm}^{2}\right)$ and upper limb skeletal muscle mass $(\mathrm{kg})$ were measured using a multifrequency bioelectrical impedance analysis device (InBody $720^{\circledR}$; Biospace, Seoul, Korea), and the relationships between VFA and the upper limb skeletal muscle mass and blood test results were investigated. Subsequently, setting the cut-off value of VFA at $100 \mathrm{~cm}^{2}$ for visceral obesity and the cut-off value of sarcopenia at $1.7 \mathrm{~kg} / \mathrm{m}^{2}$ for men and 1.2 $\mathrm{kg} / \mathrm{m}^{2}$ for women, patients were classified into normal body composition, visceral obesity, sarcopenia, and sarcopenic obesity (12), and their outcomes were observed. The European Working Group on Sarcopenia in Older People and the Asian Working Group for Sarcopenia suggested that a low muscle mass (sarcopenia) is more than 2 standard deviations (SD) below the normal young mean. Taking into account age- and disease (diabetes)-related muscle loss, values less than $1 \mathrm{SD}$ from the mean in the diabetes cases were consid- ered to indicated the presence of sarcopenia in the present study (12).

Furthermore, 50 patients with cirrhosis (27 men, 23 women; mean age $64 \pm 9$ years) who underwent multiple body composition measurements during the observation period of at least 6 months were extracted, and their initial and final measurements were compared. Specifically, the correlations between the percent changes in the blood test results and the percent changes in the body composition measurements were analyzed to identify the blood test results associated with body composition changes. Additionally, in cirrhosis patients whose body composition was measured at multiple time points, the frequencies of transitions into each body composition state during the observation period were also assessed. This investigation was conducted according to the principles expressed in the Declaration of Helsinki and it was approved by the Ethics Committee of Mie University Hospital (approval number 2028).

\section{Statistics}

The chi-square test, the Mann-Whitney $\mathrm{U}$ test, and the $t$ test were used to analyze differences between 2 groups, and Pearson's correlation coefficient or Spearman's rank correlation coefficient was used to determine any correlations between 2 groups. Multiple comparisons among the 4 groups were performed with $m \times n$ contingency tables and Scheffe's F test. The Wilcoxon signed-rank test was used to compare changes in test values. In addition, the Kaplan-Meier method was used for the survival analysis. p values of $<0.05$ were considered to be significant.

\section{Results}

\section{Relationships among cirrhosis patients' background characteristics, body composition, and blood test re- sults}

The causes of cirrhosis in the 161 patients were: hepatitis B virus (HBV) infection (9 patients), hepatitis $\mathrm{C}$ virus (HCV) infection (101 patients), alcohol (26 patients), and other (25 patients). There were 82,64 , and 15 patients with Child-Pugh severity classifications of $\mathrm{A}, \mathrm{B}$, and $\mathrm{C}$, respectively, and $59 \%$ of the patients also had coexisting HCC. Next, the relationships between the prevalence of sarcopenia and the Child-Pugh classification, ascites, and ECW ratio were evaluated. Child-Pugh class B or C cirrhotic patients tended to have a higher prevalence of sarcopenia $(19 / 64$, $30 \% ; 5 / 15,33 \%$, respectively) than class A $(16 / 82,20 \%)$, but the difference was not significant $(\mathrm{p}=0.111)$. There was no difference in the prevalence of sarcopenia in cirrhotic patients with $(14 / 55,25 \%)$ and without ascites $(26 / 106,25 \%)$. We previously reported that a relative expansion of ECW (i.e., more than 0.398) was associated with peripheral edema in patients with cirrhosis (13). Cirrhotic patients with a high ECW ratio $(>0.398)$ had a significantly $(\mathrm{p}<0.05)$ higher prevalence of sarcopenia $(25 / 73,34 \%)$ than those with a 
normal ratio $(18 / 88,17 \%)$. In this way, the prevalence of sarcopenia may increase with advancing liver disease accompanied with an expansion of ECW.

The correlations between body composition and the blood test results are shown in Table 1. Although there was a weak positive correlation between VFA and albumin, no significant correlations between the muscle mass and blood test results were observed.

\section{Classification of body composition and prognosis}

Patients were classified into the following 4 groups depending on their upper limb skeletal muscle mass and VFA: normal body composition (normal skeletal muscle mass, normal VFA); visceral obesity (normal muscle mass, VFA

Table 1. Correlations between Body Composition and Blood Test Results.

\begin{tabular}{lccc}
\hline & Albumin & ALT & T-Bil \\
\hline Skeletal muscle mass & $\mathrm{r}=0.149$ & $\mathrm{r}=0.133$ & $\mathrm{r}=0.104$ \\
(male) & $\mathrm{p}=0.149$ & $\mathrm{p}=0.200$ & $\mathrm{p}=0.314$ \\
Skeletal muscle mass & $\mathrm{r}=-0.020$ & $\mathrm{r}=-0.114$ & $\mathrm{r}=0.083$ \\
(female) & $\mathrm{p}=0.872$ & $\mathrm{p}=0.360$ & $\mathrm{p}=0.505$ \\
VFA & $\mathrm{r}=0.238$ & $\mathrm{r}=0.149$ & $\mathrm{r}=0.077$ \\
& $\mathrm{p}<0.01$ & $\mathrm{p}=0.06$ & $\mathrm{p}=0.339$ \\
\hline
\end{tabular}

VFA: visceral fat area, ALT: alanine aminotransferase, T-Bil: total bilirubin
$>100$ ); sarcopenia (decreased muscle mass, normal VFA); and sarcopenic obesity (decreased muscle mass, VFA >100). There were 60, 61, 25, and 15 patients, respectively, in each group (Table 2). Sarcopenia and sarcopenic obesity were more commonly observed in men, and sarcopenic obesity was more common observed in elderly individuals. Diabetes and other causes of cirrhosis were more common in cirrhosis patients with visceral obesity. No differences were observed in the coexistence of HCC, severity of liver disease, and serum albumin (Table 2). The groups were also compared regarding the concentrations of alpha-fetoprotein and proteins induced by vitamin $\mathrm{K}$ absence-II on admission, HCC clinical stage (Liver Cancer Study Group of Japan), initial treatment or recurrence of $\mathrm{HCC}$, details of treatment (hepatectomy, radiofrequency ablation, transarterial chemoembolization, transcatheter arterial infusion, sorafenib, or untreated), and whether or not branched-chain amino acids (BCAAs) were administered. However, there were no significant differences in any of the items (Table 2).

In the comparison of 3 groups (visceral obesity, sarcopenia, and sarcopenic obesity groups), 22 (36\%), 12 (48\%), and $10(67 \%)$ deaths were noted, respectively, during a mean observation period of 1,005 days, and the prognosis was significantly worse in the cases with sarcopenic obesity, followed by those with sarcopenia and visceral obesity, in

Table 2. Classification of Body Composition and Clinical Findings.

\begin{tabular}{|c|c|c|c|c|}
\hline & $\begin{array}{c}\text { Sarcopenic } \\
\text { obesity }(n=15)\end{array}$ & $\begin{array}{l}\text { Sarcopenia } \\
(\mathrm{n}=25)\end{array}$ & $\begin{array}{l}\text { Obesity } \\
(n=61)\end{array}$ & $\begin{array}{l}\text { Normal } \\
(n=60)\end{array}$ \\
\hline & & • & * & $\neg$ \\
\hline \multirow[t]{2}{*}{ Male/Female } & $13 / 2$ & $18 / 7$ & $35 / 26$ & $28 / 32$ \\
\hline & $\Gamma$ & $*$ & & 7 \\
\hline Age (y) & $74 \pm 4$ & $67 \pm 10$ & $\begin{array}{r}66 \pm 7 \\
* \quad 6\end{array}$ & $66 \pm 10$ \\
\hline HBV/HCV/alcohol/others & $2 / 9 / 3 / 1$ & $0 / 15 / 8 / 2$ & $\begin{array}{r}5 / 33 / 5 / 18 \\
*\end{array}$ & $1 / 40 / 6 / 13$ \\
\hline Diabetes mellitus (present/absent) & $3 / 12$ & $10 / 15$ & $32 / 29$ & $14 / 46$ \\
\hline HCC (present/absent) & $9 / 6$ & $16 / 9$ & $39 / 22$ & $31 / 29$ \\
\hline $\operatorname{AFP}(\mathrm{ng} / \mathrm{mL})$ & $29 \pm 47$ & $82 \pm 184$ & $431 \pm 1447$ & $558 \pm 2974$ \\
\hline PIVKA-II (mAU/mL) & $53 \pm 75$ & $3111 \pm 8908$ & $296 \pm 828$ & $1101 \pm 3153$ \\
\hline HCC clinical stage (I/II/III/IVA/IVB & B) $1 / 4 / 4 / 0 / 0$ & $1 / 6 / 6 / 2 / 1$ & $4 / 18 / 13 / 2 / 2$ & $4 / 10 / 10 / 4 / 3$ \\
\hline Initial treatment/recurrence & $1 / 8$ & $6 / 10$ & $15 / 24$ & $8 / 23$ \\
\hline Hepatectomy/RFA/TACE+RFA/TAC & C $0 / 1 / 3 / 3$ & $1 / 1 / 2 / 2$ & $0 / 3 / 17 / 6$ & $2 / 1 / 7 / 5$ \\
\hline E/TAI/Sorafenib/untreated/others & $/ 2 / 0 / 0 / 0$ & $/ 2 / 0 / 2 / 6$ & $/ 5 / 0 / 0 / 8$ & $/ 9 / 1 / 1 / 5$ \\
\hline Child-Pugh score & $7.0 \pm 1.6$ & $7.4 \pm 2.0$ & $6.3 \pm 1.5$ & $7.1 \pm 1.7$ \\
\hline Child-Pugh class $(\mathrm{A} / \mathrm{B} / \mathrm{C})$ & $8 / 5 / 2$ & $8 / 14 / 3$ & $38 / 20 / 3$ & $28 / 25 / 7$ \\
\hline Albumin (g/dL) & $3.3 \pm 0.5$ & $3.1 \pm 0.5$ & $3.4 \pm 0.6$ & $3.2 \pm 0.6$ \\
\hline Use of BCAAs (+/-) & $10 / 5$ & $16 / 9$ & $34 / 27$ & $33 / 27$ \\
\hline BCAA granules/BCAA mixtures & $6 / 4$ & $8 / 8$ & $19 / 15$ & $16 / 17$ \\
\hline Survival/death (\%) & $5 / 10(67 \%)$ & $13 / 12(48 \%)$ & $39 / 22(36 \%)$ & $31 / 29(48 \%)$ \\
\hline Cause of death (HCC/liver & $0 / 3$ & $2 / 3$ & $8 / 1$ & $0 / 3$ \\
\hline failure/renal failure/others) & $/ 0 / 7$ & $/ 0 / 7$ & $/ 1 / 12$ & $/ 0 / 16$ \\
\hline
\end{tabular}


A

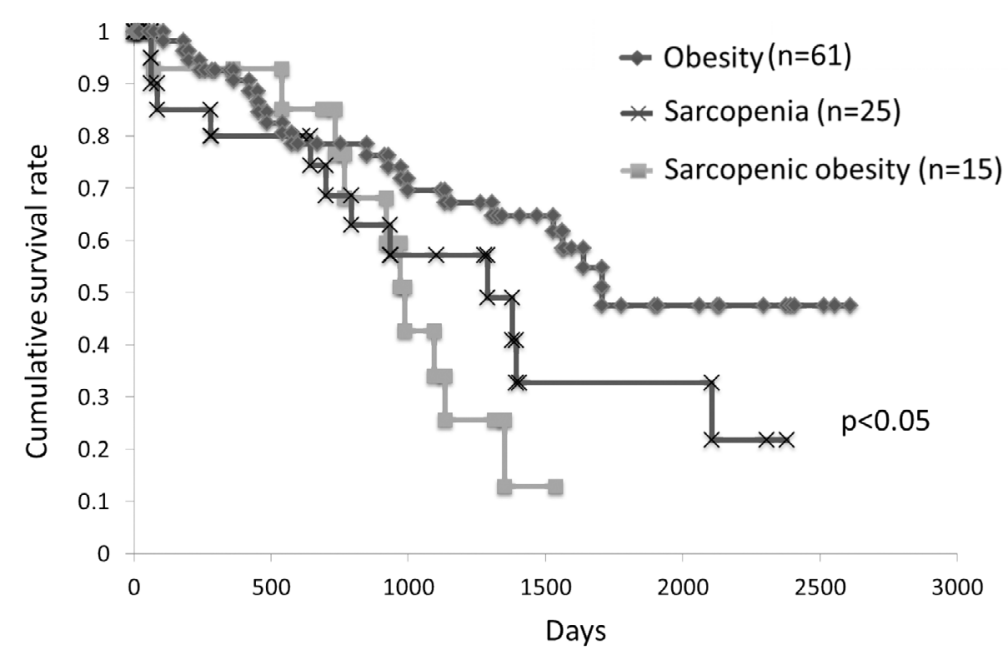

B

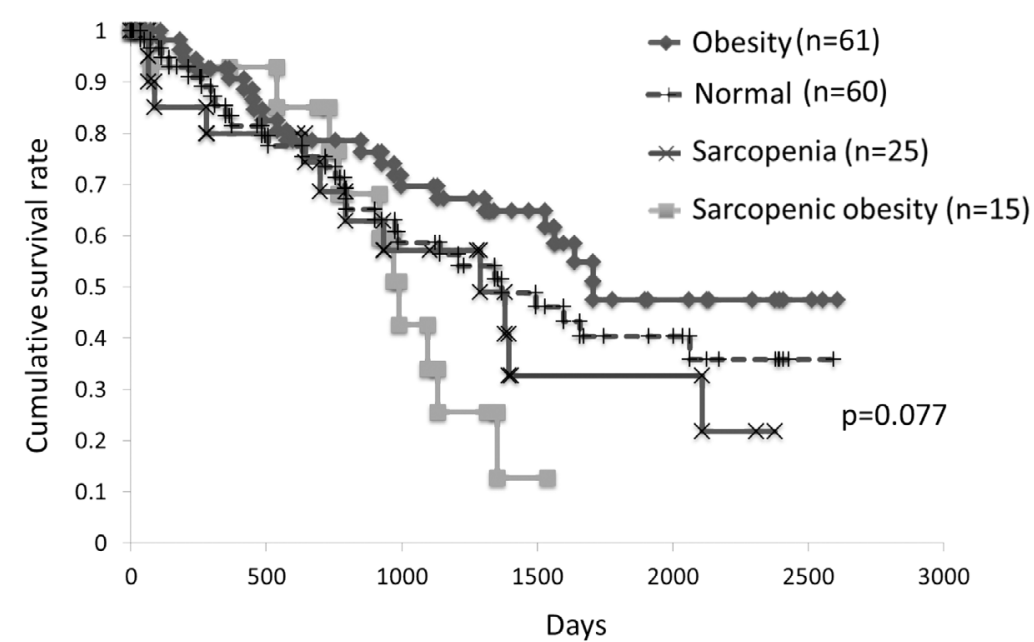

C

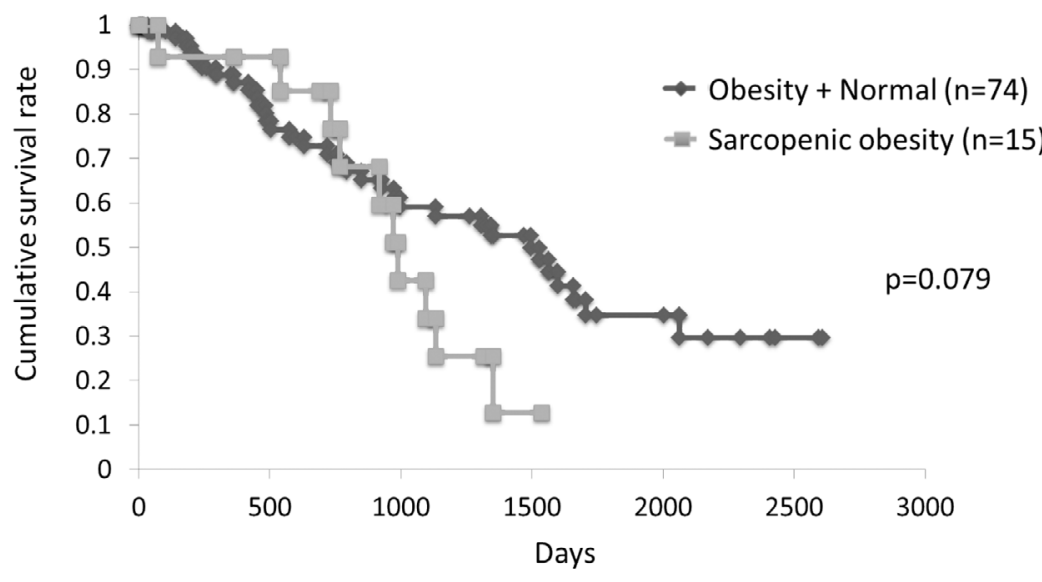

Figure 1. A: Classification of body composition and prognosis. The prognosis is significantly worse in patients with sarcopenic obesity, followed by sarcopenia and visceral obesity, in that order $(\mathbf{p}<0.05)$. B: Classification of body composition and prognosis. The prognosis is poor for sarcopenic obesity, followed by sarcopenia, normal body composition, and visceral obesity, in that order $(p=0.077)$. $\mathrm{C}$ : Classification of body composition and the prognosis limited to elderly (aged $\geq 64$ years) subjects. The prognosis is poorer for sarcopenic obesity than for a normal body composition plus visceral obesity

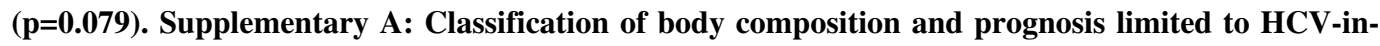
fected patients. There is no association between body composition and survival. Supplementary B: If sarcopenia is defined as an appendicular skeletal muscle mass according to the definition of the Asian Working Group for Sarcopenia, i.e., $7.0 \mathrm{~kg} / \mathrm{m}^{2}$ in men and $5.7 \mathrm{~kg} / \mathrm{m}^{2}$ in women, there are 23, 37, 53, and 48 patients with sarcopenic obesity, sarcopenia, obesity, and normal body composition, respectively and there was no association between body composition and sarcopenia. 

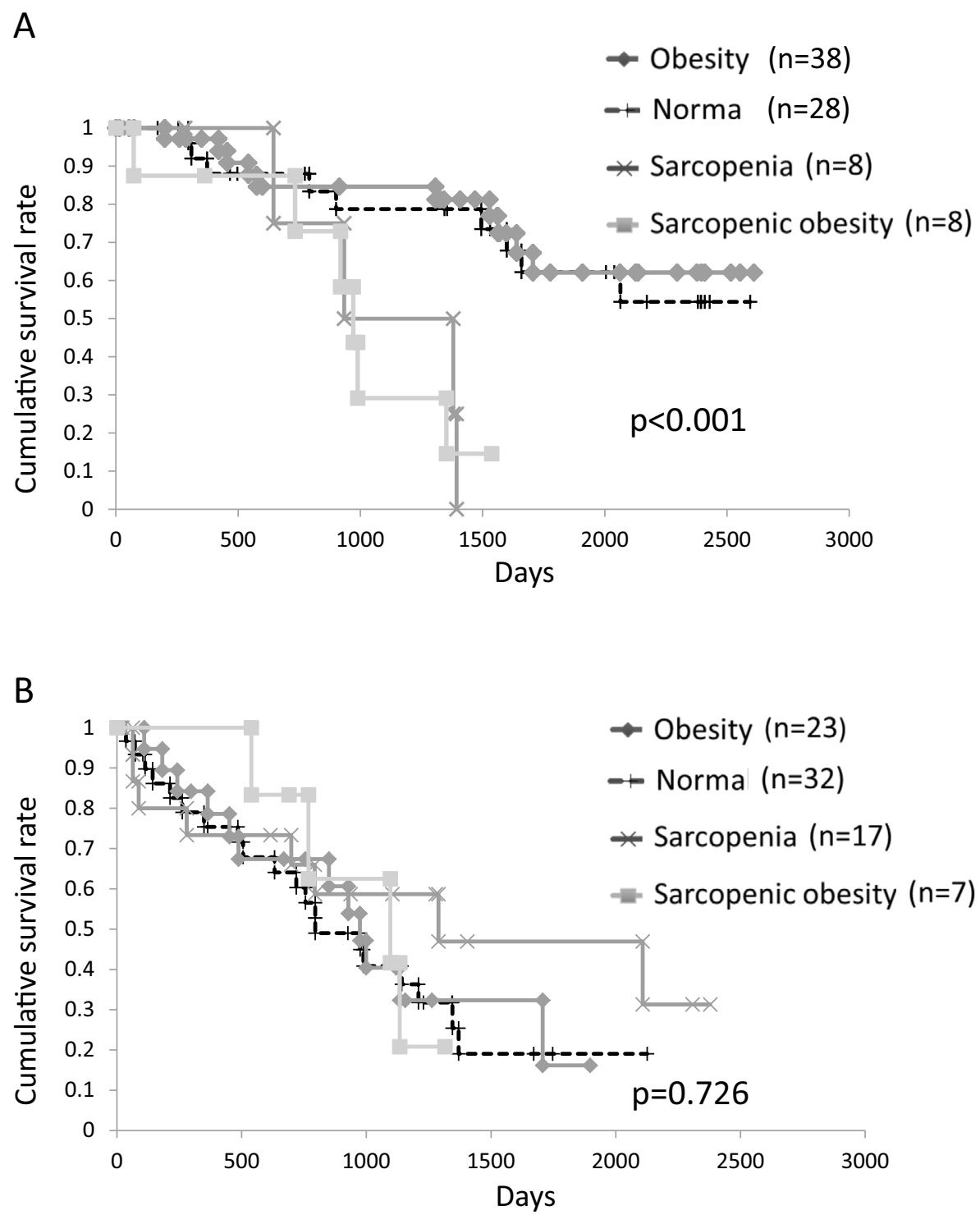

Figure 2. A: Classification of body composition and prognosis limited to Child-Pugh class A cases. Patients with sarcopenia or sarcopenic obesity have a poorer prognosis than with those with a normal body composition or visceral obesity $(\mathbf{p}<\mathbf{0 . 0 0 1})$. B: Classification of body composition and prognosis limited to Child-Pugh class $\mathrm{B}+\mathrm{C}$. There is no association between the body composition and survival.

that order $(\mathrm{p}<0.05)($ Fig. 1A). Adding the normal body composition group to this comparison, the 4-group comparison showed that the prognosis was poor in sarcopenic obesity, followed by sarcopenia, normal body composition, and visceral obesity, in that order ( $\mathrm{p}=0.077$ ) (Fig. 1B). In the subgroup analysis using the Child-Pugh classification, patients with sarcopenia or sarcopenic obesity had a poor prognosis in the subset of patients classified as Child-Pugh class A (Fig. 2A), but no differences were seen among the class B and $\mathrm{C}$ patients (Fig. 2B). As shown in Fig. 2A, survival was similar for patients with normal body composition and patients with visceral obesity. In HCV-infected patients ( $\mathrm{n}=$ 101), there was no association between body composition and survival (Supplementary Fig. 1A). The low number of cases may have affected this result. Since sarcopenic obesity was more common in elderly subjects, additional statistical analysis limited to elderly (aged $\geq 64$ years) subjects was performed. The mean age was $74 \pm 4$ years for patients with sarcopenic obesity and $71 \pm 5$ years for patients with normal a body composition plus visceral obesity; this was not significantly different $(\mathrm{p}=0.063)$. This comparison also showed that the prognosis tended to be poorer in cases with sarcopenic obesity $(n=15)$ than in those with a normal body composition plus visceral obesity $(n=74)(p=0.079)$ (Fig. 1C).

\section{Time-dependent changes in body composition and correlations between the percent changes in body composition and blood test measurements}

Table 3 shows body composition at the initial and final measurements, as well as changes in clinical findings. Regarding the spacing between the initial and final examinations, the shortest was 181 days, and the longest was 3,061 days, with a median of 912 days. During the observation period, an exacerbation of the severity of liver disease was ob- 
Table 3. Time-dependent Changes in Clinical Findings and Body Composition.

\begin{tabular}{|c|c|c|}
\hline & Initial & Final \\
\hline \multicolumn{3}{|l|}{ Clinical finding $(\mathrm{n}=50)$} \\
\hline Age (y) & $64 \pm 9$ & $67 \pm 8^{* *}$ \\
\hline $\mathrm{HBV} / \mathrm{HCV} / \mathrm{Alcohol} /$ Others & $3 / 29 / 9 / 9$ & \\
\hline Diabetes mellitus & $24(48 \%)$ & $24(48 \%)$ \\
\hline $\mathrm{HCC}$ & $21(42 \%)$ & $23(46 \%)$ \\
\hline Child-Pugh score & $5.7 \pm 0.5$ & $6.0 \pm 1.3^{*}$ \\
\hline \multicolumn{3}{|l|}{ Body composition $(n=50)$} \\
\hline $\operatorname{VFA}\left(\mathrm{cm}^{2}\right)$ & $110 \pm 38$ & $111 \pm 39$ \\
\hline Skeletal muscle mass $\left(\mathrm{kg} / \mathrm{m}^{2}\right)$ & $1.76 \pm 0.39$ & $1.78 \pm 0.41$ \\
\hline Sarcopenic obesity & $3(6 \%)$ & $2(4 \%)$ \\
\hline Sarcopenia & $5(10 \%)$ & $6(12 \%)$ \\
\hline Obesity & $27(54 \%)$ & $28(56 \%)$ \\
\hline Normal & $15(30 \%)$ & $14(28 \%)$ \\
\hline \multicolumn{3}{|l|}{ Sarcopenic obesity $(n=3)$} \\
\hline $\operatorname{VFA}\left(\mathrm{cm}^{2}\right)$ & $109 \pm 5$ & $112 \pm 32$ \\
\hline Skeletal muscle mass $\left(\mathrm{kg} / \mathrm{m}^{2}\right)$ & $1.50 \pm 0.27$ & $1.58 \pm 0.43$ \\
\hline \multicolumn{3}{|l|}{ Sarcopenia $(n=5)$} \\
\hline VFA $\left(\mathrm{cm}^{2}\right)$ & $70 \pm 27$ & $81 \pm 17$ \\
\hline Skeletal muscle mass $\left(\mathrm{kg} / \mathrm{m}^{2}\right)$ & $1.37 \pm 0.22$ & $1.50 \pm 0.21 *$ \\
\hline \multicolumn{3}{|l|}{ Obesity $(n=27)$} \\
\hline $\operatorname{VFA}\left(\mathrm{cm}^{2}\right)$ & $135 \pm 29$ & $131 \pm 35$ \\
\hline Skeletal muscle mass $\left(\mathrm{kg} / \mathrm{m}^{2}\right)$ & $1.87 \pm 0.37$ & $1.87 \pm 0.39$ \\
\hline \multicolumn{3}{|l|}{ Normal $(n=15)$} \\
\hline $\operatorname{VFA}\left(\mathrm{cm}^{2}\right)$ & $75 \pm 15$ & $83 \pm 30$ \\
\hline Skeletal muscle mass $\left(\mathrm{kg} / \mathrm{m}^{2}\right)$ & $1.74 \pm 0.42$ & $1.74 \pm 0.46$ \\
\hline
\end{tabular}

served. Regarding body composition, only sarcopenic patients demonstrated an increase in muscle mass (Table 3). Unfortunately, the dietary habits and daily physical activity data of the patients were unavailable. Therefore, the association between the instructions for nutrition and exercise and the changes in body composition could not be evaluated. In the present study, the ratio of obese patients did not significantly decrease. Restriction of activities during frequent hospitalization, physical inactivity, and enteral nutritional support including BCAA-enriched mixtures and/or night-time light meals might also have affected the results. The correlations between percent changes (final-initial/initial) in body composition and in blood test results were examined, and a significantly positive correlation was observed between the percent change in the skeletal muscle mass and VFA and the percent change in the serum albumin level (Fig. 3, Table 4). No other items showed any significant correlations, and it was found that the skeletal muscle mass fluctuated in parallel with the albumin levels. Moreover, in terms of physical measurements, the BMI was also assessed, but it did not demonstrate any association with the blood test results (data not shown.)

\section{Transitions in body composition}

During the observation period, $\geq 3$ patients had transitions of body composition with each of the following combinations: normal body composition to visceral obesity, visceral obesity to normal body composition, normal body composition to sarcopenia, and visceral obesity to sarcopenic obesity. It was postulated that the transition between a normal composition and visceral obesity can occur in either direc-
Table 4. Correlation between Percent Changes in Body Composition and Blood Test Measurements.

\begin{tabular}{lccc}
\hline & $\begin{array}{c}\text { Change in } \\
\text { albumin }\end{array}$ & $\begin{array}{c}\text { Change in } \\
\text { ALT }\end{array}$ & $\begin{array}{c}\text { Change in } \\
\text { T-Bil }\end{array}$ \\
\hline Change in VFA & $\mathrm{r}=0.351$ & $\mathrm{r}=0.026$ & $\mathrm{r}=0.028$ \\
& $\mathrm{p}<0.05$ & $\mathrm{p}=0.857$ & $\mathrm{p}=0.844$ \\
Change in skeletal & $\mathrm{r}=0.542$ & $\mathrm{r}=-0.159$ & $\mathrm{r}=-0.135$ \\
muscle mass & $\mathrm{p}<0.001$ & $\mathrm{p}=0.270$ & $\mathrm{p}=0.351$ \\
\hline
\end{tabular}

VFA: visceral fat area, ALT: alanine aminotransferase, T-Bil: total bilirubin

tion depending on energy intake and physical activity, and that transitions in normal body composition to sarcopenia and visceral obesity to sarcopenic obesity accompany a progression of cirrhosis.

\section{Discussion}

The present investigation re-confirmed that there is a strong relationship between the prognosis and body composition, especially between the prognosis and sarcopenia in patients with cirrhosis. A new finding from this study is that patients with sarcopenic obesity also had a poor prognosis. These findings partially corroborate the findings of a report that measured the skeletal muscle mass and visceral fat mass with CT and analyzed the correlation with the prognosis after liver resection for cancer, thus concluding that sarcopenia is a poor prognostic factor (8). Specifically, the idea is that, while sarcopenia is a common abnormality of body composition in advanced cirrhosis cases, the visceral fat mass can decrease through undernutrition (cachexia) or increase through decreased physical activity (sarcopenic obesity) (5). The present study did not show a favorable prognosis in cirrhosis patients with visceral obesity. Studies in hemodialysis patients showed that the prognosis is poor in low body weight patients and satisfactory in overweight patients $(14,15)$. We previously reported that survival was worse with a decreased VFA $\left(<70 \mathrm{~cm}^{2}\right)(16)$. In the present study, there was also an association between VFA and the serum albumin level. A better nutritional status may therefore explain the association between visceral obesity with a better survival in cirrhotic patients. Further investigation of this issue will thus be needed.

In the present study, the longitudinal measurements of body composition and blood test items demonstrated that albumin was the only blood test measurement that showed a correlation with changes in muscle mass. In addition, visceral fat mass and albumin showed a mild positive correlation with each other, and the changes over time paralleled each other. It is a well-known fact that a lean body weight is the nutritional factor that is most relevant to prognosis, and that the primary components of lean mass are muscle protein and visceral proteins such as albumin. The present longitudinal study re-confirmed this fact, indicating that it would be essential to provide nutritional guidance in the actual clinical situation, bearing in mind that the maintenance 
A

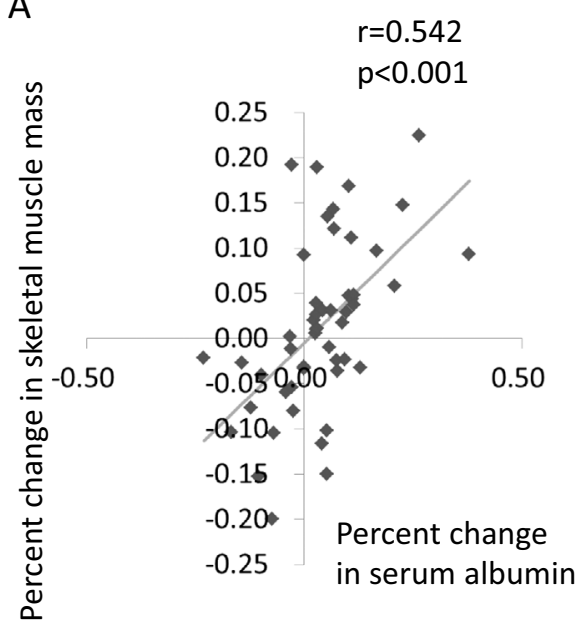

B

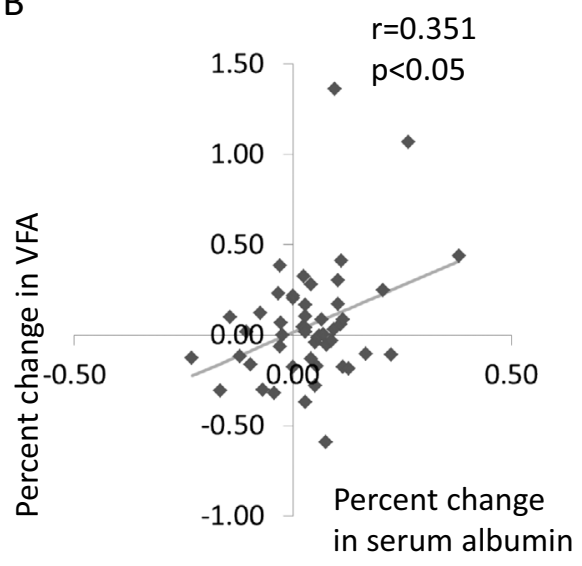

Figure 3. A significant positive correlation is observed between percent change in skeletal muscle mass and the percent change in serum albumin (A). A significant positive correlation is observed between the percent change in VFA and the percent change in serum albumin (B). VFA: visceral fat area

of the serum albumin levels leads to the retention of skeletal muscle.

As mentioned previously, sarcopenia is a common feature of advanced cirrhosis, and the visceral fat mass can decrease or increase depending on the circumstances (5). The present longitudinal study also supports this, as shown in the results, which indicate that the stage preceding sarcopenia is mainly a normal body composition, and the stage preceding sarcopenic obesity is mainly visceral obesity. Although the causes for this and the suppression of secondary sarcopenia that accompanies cirrhosis are unknown (17), at the present time, nutritional therapy, such as high-protein meals that are intended to maintain albumin, the administration of BCAA formulations, and the night-time administration of light meals, is suggested to be helpful $(18,19)$.

This study is associated with several limitations. In the present study, the cut-off value of sarcopenia was defined by the upper limb skeletal muscle mass. The Asian Working Group for Sarcopenia suggested that the cut-off value is 7.0 $\mathrm{kg} / \mathrm{m}^{2}$ in men and $5.7 \mathrm{~kg} / \mathrm{m}^{2}$ in women using the appendicular skeletal muscle mass (20). According to this definition, there were $23,37,53$, and 48 patients with sarcopenic obesity, sarcopenia, obesity, and normal body composition, respectively, and there was no association between body composition and sarcopenia (Supplementary Fig. 1B and Supplementary Table 1). Another issue associated with the present study is that a multi-frequency bioelectrical impedance analysis device was used to measure the body composition. This method is often used clinically due to its convenience and superior reproducibility, and it is also recommended for detailed examinations of undernutrition in cirrhosis (recommendation level B) in the 2006 ESPEN guideline (11). However, caution is necessary in diseases that lead to an increased amount of body fluids, such as cirrhosis, since such measurements may be overestimated (21). In order to mini- mize this issue, upper limb muscle mass measurements were used for our evaluations (12). The upper limb is widely used for traditional physical measurements and it is quite suitable for this purpose as well. Indeed, more sarcopenia was detected even in advanced liver disease with an expansion of ECW. While VFA measurements using the impedance method tend to be problematic, based on their strong correlation with VFA measured by CT and previous findings in chronic diseases, we believe it is a fully sufficient biomarker for clinical application (22-24).

Sarcopenia and sarcopenic obesity are poor prognostic factors in patients with cirrhosis. To improve the prognosis of cirrhosis, the skeletal muscle mass and VFA monitoring, as well as the administration of appropriate nutritional therapy, including the maintenance of albumin, is recommended.

The authors state that they have no Conflict of Interest (COI).

\section{References}

1. Verhage TL, Heijdra Y, Molema J, Vercoulen J, Dekhuijzen R. Associations of muscle depletion with health status. Another gender difference in COPD? Clin Nutr 30: 332-338, 2011.

2. Lieffers JR, Bathe OF, Fassbender $K$, Winget $M$, Baracos VE. Sarcopenia is associated with postoperative infection and delayed recovery from colorectal cancer resection surgery. $\mathrm{Br} \mathrm{J}$ Cancer 107: 931-936, 2012.

3. Walsmith $\mathbf{J}$, Roubenoff R. Cachexia in rheumatoid arthritis. Int $\mathbf{J}$ Cardiol 85: 89-99, 2002.

4. Durand F, Buyse S, Francoz C, et al. Prognostic value of muscle atrophy in cirrhosis using psoas muscle thickness on computed tomography. J Hepatol 60: 1151-1157, 2014.

5. Biolo G, Cederholm T, Muscaritoli M. Muscle contractile and metabolic dysfunction is a common feature of sarcopenia of aging and chronic diseases: from sarcopenic obesity to cachexia. Clin Nutr 33: 737-748, 2014.

6. Lu CW, Yang KC, Chang HH, Lee LT, Chen CY, Huang KC. Sar- 
copenic obesity is closely associated with metabolic syndrome. Obes Res Clin Pract 7: e301-e307, 2013.

7. Lim S, Kim JH, Yoon JW, et al. Sarcopenic obesity: prevalence and association with metabolic syndrome in the Korean Longitudinal Study on Health and Aging (KLoSHA). Diabetes Care 33: 1652-1654, 2010

8. Itoh S, Shirabe K, Matsumoto Y, et al. Effect of body composition on outcomes after hepatic resection for hepatocellular carcinoma. Ann Surg Oncol 21: 3063-3068, 2014.

9. Amodio $\mathrm{P}$, Bemeur $\mathrm{C}$, Butterworth $\mathrm{R}$, et al. The nutritional management of hepatic encephalopathy in patients with cirrhosis: International Society for Hepatic Encephalopathy and Nitrogen Metabolism Consensus. Hepatology 58: 325-336, 2013.

10. Moctezuma-Velázquez C, García-Juárez I, Soto-Solís R, Hernández-Cortés J, Torre A. Nutritional assessment and treatment of patients with liver cirrhosis. Nutrition 29: 1279-1285, 2013.

11. Plauth M, Cabré E, Riggio O, et al. ESPEN Guidelines on Enteral Nutrition: Liver disease. Clin Nutr 25: 285-294, 2006.

12. Iwasa M, Hara N, Terasaka E, et al. Evaluation and prognosis of sarcopenia using impedance analysis in patients with liver cirrhosis. Hepatol Res 44: E316-E317, 2014.

13. Hara N, Iwasa M, Iwata $K$, et al. Value of the extracellular water ratio for assessment of cirrhotic patients with and without ascites. Hepatol Res 39: 1072-1079, 2009.

14. Kalantar-Zadeh K, Abbott KC, Salahudeen AK, Kilpatrick RD, Horwich TB. Survival advantages of obesity in dialysis patients. Am J Clin Nutr 81: 543-554, 2005.

15. Tsirpanlis G, Petrihou C, Savva L, et al. Body mass index and mortality in CKD: explaining the obesity paradox. Am J Kidney Dis 51: 533, 2008.
16. Iwasa M, Hara N, Hattori A, et al. Body composition in patients with liver cirrhosis: survival prognosis and sex differences. Nutr Diet 72: 299-301, 2015.

17. Qiu J, Thapaliya S, Runkana A, et al. Hyperammonemia in cirrhosis induces transcriptional regulation of myostatin by an NF- $\mathrm{kB}-$ mediated mechanism. Proc Natl Acad Sci USA 110: 18162-18167, 2013.

18. Holecek M. Three targets of branched-chain amino acid supplementation in the treatment of liver disease. Nutrition 26: 482-490, 2010.

19. Nakaya $Y$, Shimohata $T$, Haraguchi $S$, et al. Severe catabolic state after an overnight fast in patients with chronic renal failure. Nutrition 27: 329-332, 2011.

20. Chen LK, Liu LK, Woo J, et al. Sarcopenia in Asia: consensus report of the Asian Working Group for Sarcopenia. J Am Med Dir Assoc 15: 95-101, 2014.

21. Kyle UG, Bosaeus I, De Lorenzo AD, et al; ESPEN. Bioelectrical impedance analysis-part II: utilization in clinical practice. Clin Nutr 23: 1430-1453, 2004.

22. Iwasa $\mathrm{M}$, Mifuji-Moroka $\mathrm{R}$, Hara $\mathrm{N}$, et al. Visceral fat volume predicts new-onset type 2 diabetes in patients with chronic hepatitis C. Diabetes Res Clin Pract 94: 468-470, 2011.

23. Iwasa M, Hara N, Iwata $\mathrm{K}$, et al. Restriction of calorie and iron intake results in reduction of visceral fat and serum alanine aminotransferase and ferritin levels in patients with chronic liver disease. Hepatol Res 40: 1188-1194, 2010.

24. Iwata K, Iwasa M, Nakatani T, et al. Seasonal variation in visceral fat and blood HbAlc in people with type 2 diabetes. Diabetes Res Clin Pract 96: e53-e54, 2012.

(C) 2016 The Japanese Society of Internal Medicine http://www.naika.or.jp/imonline/index.html 